\title{
O Efeito Termoiônico: Uma Nova Proposta Experimental
}

\author{
E.F. de Lima, M. Foschini e M. Magini ${ }^{\dagger}$ \\ Instituto de Física de São Carlos, USP \\ Caixa Postal 369, CEP.:13560 - 970, S.P., Brazil \\ ${ }^{\dagger}$ Autor de Contato: magini@if.sc.usp.br
}

Recebido em 11 de Abril de 2001. Aceito em 30 de Outubro de 2001.

\begin{abstract}
O efeito termoiônico desempenha um importante papel no desenvolvimento da ciência e da compreensão das propriedades da matéria. Aqui é feita uma proposta simples visando o estudo deste efeito com o uso de uma lâmpada de carro de dois filamentos. Através da experiência, foi possível determinar a equação de Richardson - Dushman, a Lei de child e observar propriedades de retificação. Estes resultados mostram que, através de uma proposta simples, é possível estudar o efeito termoiônico em toda a sua riqueza física.
\end{abstract}

The thermoionic effect plays an important role in development of science and understanding of matter properties. In this present an experiment was performed to study this effect using a simple car lamp with two filaments. From this experiment we determined the Richardson - Dushman equation, Child's Law and measured rectification properties. From these results we conclude through this simple experiment was possible to study the thermoionic effect in its entirety.

\section{Introdução}

O efeito termoiônico é definido como a emissão de elétrons por uma superfície metálica aquecida. Os primeiros sinais do fenômeno foram observados em meados do século XVIII por Charles DuFay [1, 2], que notou que um um gás conduzia eletricidade quando colocado próximo a um sólido aquecido. Após as observações de DuFay, em 1853 o físico francês Edmund Becquerel mostrou que é possível produzir corrente elétrica a partir de um potencial gerado entre dois eletrodos de platina quente com ar aquecido entre estes [2]. Finalmente, em 1883, Thomas A. Edison verificou que elétrons são emitidos quando um metal é aquecido $[3,4,5]$.

Podemos compreender o efeito termoiônico de uma maneira simples. Ao fornecer energia térmica a um material, seus elétrons ganham energia cinética. Haverá portanto a emissão desses elétrons se sua energia for suficiente para superar a barreira de potencial da superfície do material.

É possível associar aos elétrons que saem do material uma densidade de corrente a que depende das características do material. Uma dessas características é a função trabalho, que é a energia mínima que se deve fornecer a um elétron para removê-lo do metal, por exemplo, a função trabalho do Tungstênio é cerca de $4,52 \mathrm{eV}$ [4].
A dedução da forma da densidade de corrente para efeito termoiônico requer um conhecimento de Mecânica Quântica Estatística. Como a incursão aprofundada nesse assunto está fora das pretensões deste trabalho, nos limitaremos em apresentar a fórmula da densidade de corrente, proposta inicialmente por Richardson em 1916 e corrigida por Dushman em 1930 $[1,2]$, dada por:

$$
J_{0}=A T^{2} e^{(-\Psi) / k T}\left(\text { amperes } / \mathrm{cm}^{2}\right) .
$$

onde $A$ é uma constante que é igual a 120,4 amperes $/ \mathrm{cm}^{2}, k$ é a constante de Boltzman, $T$ é a temperatura em graus Kelvin, $\Psi$ é a função trabalho do material em elétron-volts.

As experiências envolvendo a observação deste efeito são realizadas comumente em válvulas, que são dispositivos constituídos basicamente de um tubo onde se faz vácuo com dois eletrodos internos. O efeito é verificado através da geração de uma diferença de potencial elétrico entre os eletrodos, sendo o terminal negativo chamado de catodo e o positivo, de anodo. Aquecendose suficientemente o catodo, observar-se-á uma corrente elétrica no terminal positivo (anodo).

Para se obter uma expressão para a corrente coletada pelo anodo, faz-se necessária a análise de toda a dinâmica que vai desde da emissão do elétron pelo catodo até a sua absorção pelo anodo. Portanto, fa- 
tores como a geometria do catodo, a forma do potencial elétrico entre o catodo e o anodo, como também a presença de outros elétrons emitidos devem ser levados em consideração. Para eletrodos planos a densidade de corrente é dada pela lei de Child [4, 5], segundo a equação:

$$
J=B V^{3 / 2},
$$

onde $B$ é uma constante que depende da geometria da válvula e $V$ é a diferença de potencial entre o catodo e o anodo.

No presente trabalho apresentamos uma nova montagem para o estudo deste efeito utilizando-se uma lâmpada de carro de dois filamentos como válvula. A montagem experimental é bastante simples, possibilitando seu uso para fins didáticos, tanto no ensino médio como em cursos superiores introdutórios. Exibimos também, juntamente com os resultados experimentais, uma calibração que pode ser usada como padrão nas experiências de mesma natureza.

\section{Montagem Experimental}

A experiência pode ser montada de forma simples utilizando uma lâmpada de carro comum. Existem vários tipos de lâmpada, no nosso caso utilizamos uma lâmpada $^{1}$ de duplo filamento usada em geral como lanterna e luz de freio na parte traseira do carro. $\mathrm{O}$ ponto forte da experiência é o seu baixo custo e sua facilidade de montagem. A Fig. 1 mostra esquematicamente a experiência.

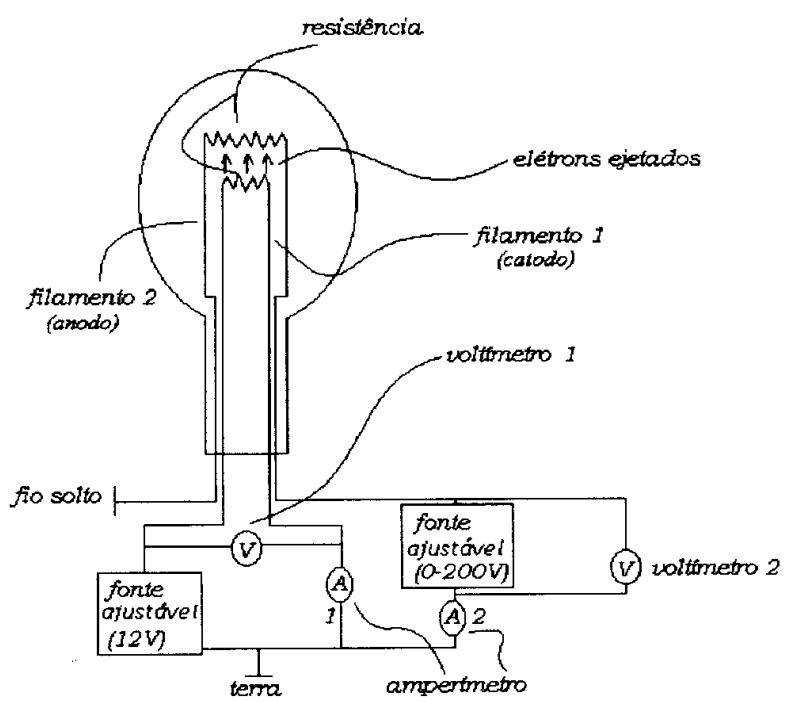

Figura 1. Esquema de montagem do efeito termoiônico que usa lâmpada de carro de dois filamentos.

O sistema consiste em:
* Duas fontes CC variáveis: uma de $12 \mathrm{~V}$ e outra de $200 \mathrm{~V}$.

* Uma fonte CA de $50 \mathrm{~V}$.

$\star$ Dois amperímetros.

$\star$ Dois voltímetros.

* Uma lâmpada de carro de dois filamentos.

* Um osciloscópio.

* Um resistor de $10 k \Omega$

A fonte de $12 \mathrm{~V}$ é utilizada para aquecer o filamento 1 por efeito Joule. Sua temperatura pode ser estimada através da potência dissipada no filamento com a ajuda da Fig. 2, na qual as medidas de temperatura foram feitas utilizando-se um pirômetro óptico. Nessa figura, assim como nas Figs. 4 e 5, foi feito um ajuste linear dos pontos experimentais representado pela reta tracejada.

Na Fig. 2, pode-se observar que, de forma aproximada, $P \sim T^{4}$, onde $P$ é a potência dissipada no filamento e $T$ é sua temperatura. Essa relação vai a favor à Lei de Stefan - Boltzmann onde $E=\sigma \times T^{4}$, sendo $\sigma$ a constante de Stefan que vale $5,64 \times 10^{-8} \mathrm{Jm}^{-2} \mathrm{~K}^{-4} \mathrm{~s}^{-1}$ [6]. A constante obtida através da Fig. 2, estimando-se que a área efetiva do eletrodo é da ordem de $10^{-5} \mathrm{~m}^{2}$, é igual a $5,6 \times 10^{-8} \mathrm{Jm}^{-2} \mathrm{~K}^{-4} \mathrm{~s}^{-1}$, que é da ordem correta de magnitude esperada para $\sigma$.

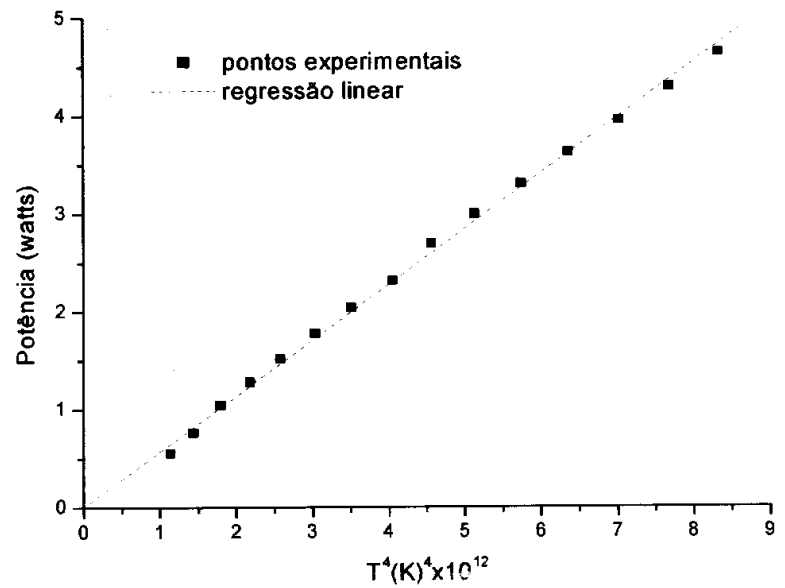

Figura 2. Relação entre temperatura e a potência dissipada no filamento aquecido - Lei de Stefan Boltzmann.

Quando aquecido, o filamento 1 emite elétrons que serão coletados pelo filamento 2 e geram uma corrente elétrica, medida no amperímetro 2. A fonte variável de $200 \mathrm{~V}$ é usada para gerar uma diferença de potencial entre os filamentos 1 e 2, a qual tem por objetivo atrair os elétrons para o anodo.

\section{Procedimentos e Resultados}

Através da montagem experimental proposta pela Fig. 1 , podemos obter a corrente versus tensão no filamento 2 para diferentes voltagens do filamento 1. Para tanto, fixa-se o valor da voltagem na fonte de $12 \mathrm{~V}$, varia-se a

\footnotetext{
${ }^{1}$ Especificações da lâmpada: OSRAM 7528 12V P21/5 W - Brasil.
} 
tensão na fonte de $200 \mathrm{~V}$ e, então, mede-se a corrente no amperímetro 2. Repetindo o procedimento para diferentes voltagens na fonte de $12 \mathrm{~V}$, obtemos a Fig. 3.

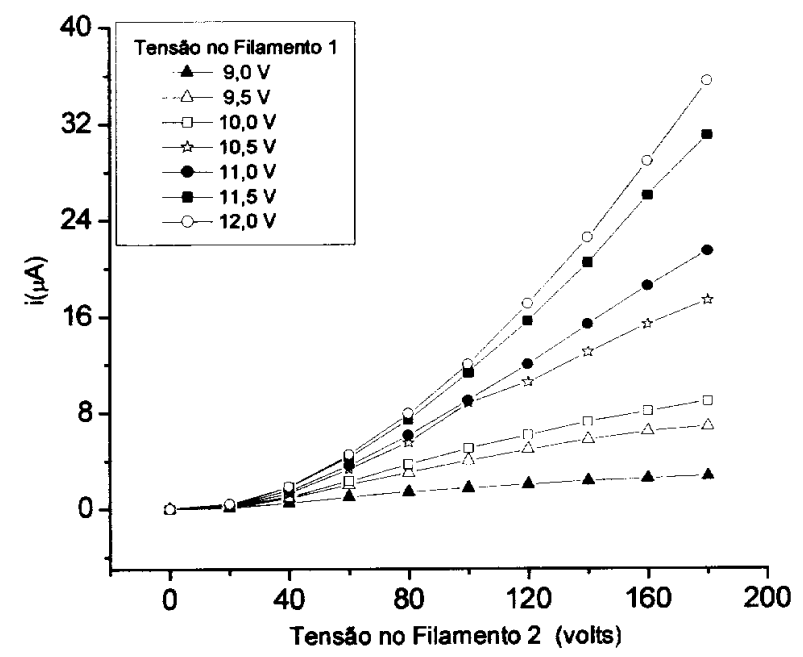

Figura 3. Corrente versus tensão no filamento 2 para diferentes voltagens do filamento 1 .

Verifica-se pela figura anterior que, para cada temperatura do catodo (voltagem do filamento 1), temos uma corrente no anodo evidenciando o efeito termoiônico.

\section{III.1 Equação de Richardson Dushman}

De acordo com a Fig. 3, observa-se que a corrente no anodo tende a um valor constante, chamada corrente de saturação, para tensões no filamento 1 de 9 a 10 volts. O mesmo não é claro para os demais valores de tensão. Nesse caso, para verificar que a tensão no anodo tende a um valor constante, teríamos que aumentar a tensão no filamento 2 acima de $200 \mathrm{~V}$, o que está acima das especificações da lâmpada utilizada. Porém, ainda podemos fazer algumas estimativas se considerarmos que os valores máximos da corrente da Fig. 3 estão próximos dos valores de saturação. Pois, quando é atingida a corrente de saturação, praticamente todos os elétrons emitidos pelo catodo são coletados no anodo, o que nos permite utilizar a equação de Richardson Dushman, eq.(1), para estimar a função trabalho do catodo.

A Fig. $4, \ln \frac{i}{T^{2}}$ versus $T^{-1}$, foi construída usando-se os valores máximos da corrente do anodo para os diferentes valores de tensão (temperatura) do catodo. Através do coeficiente angular da reta, estimamos o valor da função trabalho do catodo em aproximadamente $3,6 \mathrm{eV}$.

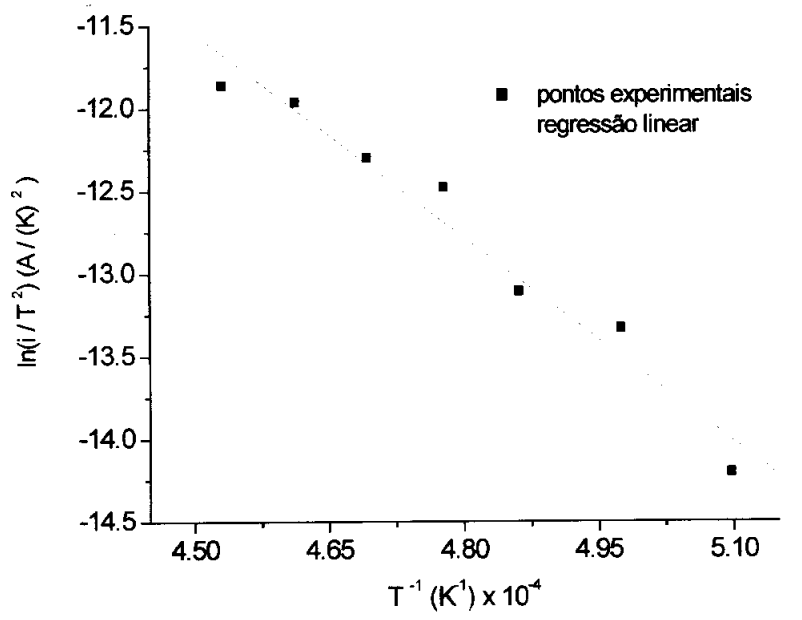

Figura 4. Determinação da função trabalho - Equação de Richardson Dushman.

\section{III.2 Lei de Child}

Como foi dito anteriormente, temos que levar em consideração, para o cálculo da densidade de corrente no anodo, a dinâmica entre a emissão e a absorção dos elétrons, assim como a geometria dos eletrodos. $\mathrm{Na}$ Fig. 5 estão os valores da corrente versus o potencial $(V)$ no anodo em escala logarítmica, obtidos para o valor da tensão (temperatura) no catodo de $11,5 \mathrm{~V}$. Pelo ajuste linear verificamos que o coeficiente angular da reta $(=1,55)$ é próximo do valor esperado pela $L e i$ de Child $(=1,5)$ eq.(2). O valor do coeficiente linear refere-se a $\ln (B)$ dado pela eq.(2).

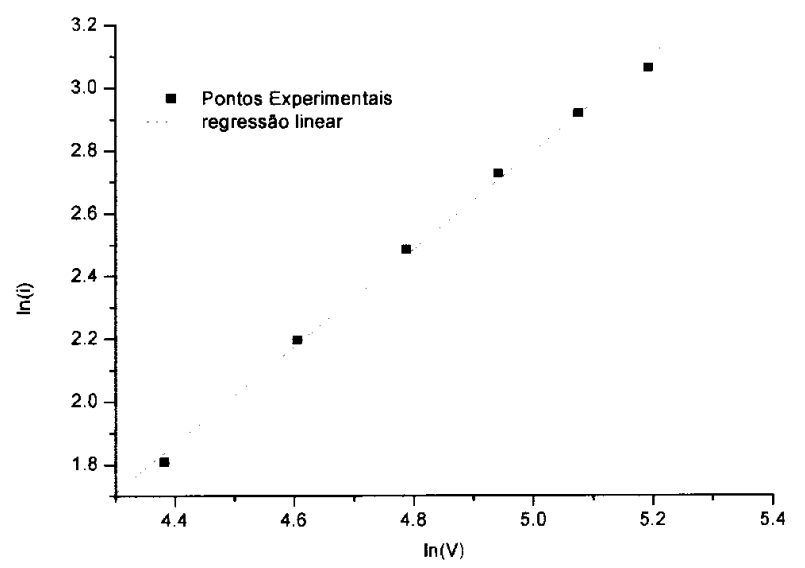

Figura 5. Lei de Child. Corrente versus potencial do anodo (escala logarítimica.)

\section{III.3 Retificação: a lâmpada como um diodo}

Modificando ligeiramente o esquema da Fig. 1, podemos verificar que a corrente na lâmpada flui em um único sentido, ou seja, funciona como um diodo. Para tanto, substituímos a fonte ajustável de $200 \mathrm{~V}$ 
pela fonte de tensão alternada em série com o resistor de $10 \mathrm{k} \Omega$, mantendo a fonte ajustável de $12 \mathrm{~V}$ ligada ao catodo. Para observar a propriedade de retificação, basta conectar o osciloscópio aos terminais do resistor e verficar que a corrente passa em um único sentido. Deve-se notar que somente a parte positiva da tensão da fonte alternada é medida no resistor. Isso acontece pois os elétrons somente atingirão o filamento 2 quando o potencial neste terminal for positivo.

Com este procedimento ilustra-se o funcionamento do diodo, cuja utilização em circuitos eletrônico é abundante. Uma possível utilização desse tipo de dispositivo é circuitos retificadores ou seja, aqueles que têm como objetivo converter um sinal alternado (que varia no tempo) em um sinal contínuo (constante). Estes circuitos estão presentes na grande maioria dos aparelhos eletrônicos, pois há a necessidade de uma tensão contínua para que este funcione.

O emprego do diodo se justifica pelo fato de que o primeiro passo para obter-se um sinal contínuo é que ele passe apenas em um único sentido. De fato, é preciso mais que diodos para construir um bom circuito retificador, contudo uma combinação de diodos, conhecida como ponte de diodos, está sempre presente nos retificadores mais tradicionais.

No nosso caso, o interesse se volta na montagem experimental. Esta vem mostrar como é possível usar a lâmpada como um diodo e entender o seu funcionamento, dada a sua importância nas diversas aplicações tecnológicas.

\section{Conclusões}

Neste trabalho, foi possível verificar o efeito termoiônico através da Fig. 3. A função trabalho obtida para filamento $(3,6 \mathrm{eV})$ através da equação de Richardson Dushman, apesar das aproximações feitas, é da mesma ordem de grandeza da função trabalho do Tungstênio $(4,52 \mathrm{eV})$ mostrando uma coerência dos resultados com o valor encontrado na literatura [4]. Observamos também que a corrente no anodo é aproximadamente proporcional a $V^{3 / 2}$, Fig. 5, para uma dada temperatura no catodo, como previsto pela Lei de Child. Além disso, observamos que a lâmpada pode funcionar como um diodo.

Apesar da simplicidade da experiência, os resultados apresentados estão de acordo com a teoria e os valores da literatura $[1,2,3]$. Como não foi feito vácuo na lâmpada, a presença de um gás na válvula modifica a dinâmica entre a emissão do elétron pelo catodo e sua absorção no anodo, justificando os desvios entre os resultados obtidos experimentalmente e aqueles encontrados na literatura. Outro fator que deve ser notado é o limite de tensão e corrente suportado por tais lâmpadas, o que nos desviou de um resultado mais preciso para a função trabalho do filamento.

Essa experiência pode ser utilizada para fins didáticos, como também em aulas demonstrativas ou de laboratório, tendo em vista seu baixo custo como também a riqueza de conceitos e fenômenos envolvidos. Desta forma, tratamos de temas corriqueiros nos cursos de física, como a emissão termoiônica, função trabalho e retificação, que podem ser abordados em cursos introdutórios, além de discutidos com mais facilidade através da experiência.

\section{References}

[1] John D. McGervey: Intorduction to Modern Physics, Academic Press, INC. Lodon, UK.

[2] The New Encyclopedia Britannica,vol. 18, página 315, MACROPAEDIA, UK.

[3] Hans Mark e N. Thomas Olson: Experiments in Modern Physics, McGraw - Hill Book Company, New York, USA.

[4] A. C. Melissimos: Experiments in Modern Physics, Academic Press INC., Florida, USA.

[5] Fred Huffman: Thermoinonic Energy Conversion, Encyclopedia of Physical Science and Technology, vol. 13, Academic Press, NY, USA.

[6] R. Eisberg e R. Resnick: Física Quântica: Átomos, Moléculas, Sólidos, Núcleos e Partículas, reimpressão, Editora Campus LTDA., RJ, Brasil. 